\title{
Surveillance for action - managing foodborne Campylobacter in New Zealand
}

\author{
Donald Campbell, a Peter van der Log ${ }^{a}$ and Steve Hathaway ${ }^{a}$ \\ Correspondences to Donald Campbell (e-mail: donald.campbell@maf.govt.nz).
}

$\mathrm{P}$ ublic health surveillance is the continuous, systematic collection, analysis and interpretation of health-related data needed for the planning, implementation and evaluation of public health practice. ${ }^{1}$ It can serve as an early warning system for impending public health emergencies; document the impact of an intervention, or track progress towards specified goals; and monitor and clarify the epidemiology of health problems, to allow priorities to be set and to inform public health policy and strategies.

In New Zealand, information gathered by the human disease surveillance system has been used to inform its well-documented, science-based Food Safety Risk Management Framework and response to an increasing national public health problem-campylobacteriosis. This paper discusses the use of surveillance data in initial prioritization, goal setting, source attribution and monitoring and review for Campylobacter infection in New Zealand.

Disease notifications provide the basis for surveillance and hence disease control in New Zealand. Health professionals and laboratories are required to inform their local Medical Officer of Health of any notifiable disease that they suspect or diagnose. These data are collated nationally, with the Ministry of Health being the responsible agency for human disease investigation and the Ministry of Agriculture and Forestry (MAF), formerly New Zealand Food Safety Authority (NZFSA), for food safety. Campylobacteriosis was made a notifiable disease in New Zealand in 1980.

The New Zealand Food Safety Risk Management Framework ensures that all aspects of internationally recognized risk analysis practice, i.e. risk assessment, risk management, risk communication and the regulatory components of monitoring and review, are brought together in a logical manner to maximize the benefits available from a risk-based approach to food safety. ${ }^{2}$ National human health surveillance activities are an important contributor to these MAF activities. To determine the effectiveness of food safety regulatory activities in consumer protection terms, food-chain monitoring and human health surveillance data are combined where possible. This may be carried out ahead of the implementation of risk management activities so as to establish baseline levels, or may follow their implementation. When setting outcomes related to consumer health, MAF wishes to be able, with a reasonable degree of certainty, to show that a change (or lack of) in disease incidence can be attributed to the organization's actions.

The successful control of foodborne disease requires knowledge about the most important sources or reservoirs as well as their principal routes. To identify and prioritize food safety interventions it is important to identify not only the fraction of incidence of human illness attributable to particular foods but also what is attributable to other sources such as environmental exposure, direct animal contact and human-tohuman exchange. ${ }^{3}$ Attribution of human foodborne diseases to source can be achieved using different methods but all depend on robust disease surveillance data. The ultimate goal is to partition the burden of disease caused by a pathogen to specific food commodities.

New Zealand has high population disease rates of several potentially foodborne diseases, especially campylobacteriosis. ${ }^{4}$ The incidence rose steadily from the mid-1980s to a peak in 2006 with 15873 cases notified (384 cases per 100000 population), the highest reported rate internationally for this disease. ${ }^{5}$ Sporadic and outbreak surveillance data, epidemiological studies, expert elicitation and microbiological (genotypic) source attribution approaches have been used to estimate

\footnotetext{
Ministry of Agriculture and Forestry, Wellington, New Zealand. Submitted: 29 March 2012; Published: 22 June 2012 doi: 10.5365/wpsar.2012.3.2.001
} 
the contribution of food and environmental sources to the incidence of campylobacteriosis. ${ }^{6-8}$ All have demonstrated that $>50 \%$ of sporadic cases were attributable to poultry. Risk ranking, where different pathogens are graded against each other, has also shown that Camplyocbacter accounts for the greatest proportion of the overall burden of disease in New Zealand.${ }^{9}$ These findings gave direction to the implementation of the MAF Campylobacter Strategies.

From 2007, the then NZFSA and the poultry industry introduced a range of regulatory and voluntary measures aimed at reducing levels of Campylobacter on fresh chicken meat. From April 2007, poultry processors were required to report Campylobacter contamination levels at the end of primary processing to the MAFmanaged National Microbiological Database (NMD); in April 2008, mandatory Campylobacter performance targets based on enumerated levels commenced; not more than six samples from 45 collected will have $>6000$ colony forming units per carcass. ${ }^{10}$ If processing plants exceed the six-sample limit, progressively greater sanctions are applied that can ultimately result in plant closure. Following the introduction of these controls, there were 9000 fewer notified cases of campylobacteriosis with 500 fewer hospitalizations in 2008 compared to $2006 .^{4}$

One of the public health goals set by MAF for the five-year period 2008-2012 was a 50\% reduction in the domestically acquired foodborne component of campylobacteriosis. To deliver this challenging target, a thermotolerant Campylobacer control strategy was developed. To scrutinize success of the strategy and progress against the public health goals, a monitoring system using surveillance data was developed. ${ }^{4}$ The statistics used are the annual (calendar year) number (per 100000 mid-year population estimate) of notified cases with the baseline year being the average of 2004-2007. The measurements are adjusted for the proportion of cases reported as having travelled overseas during likely incubation period and for the proportion of disease estimated to be due to foodborne transmission based on expert elicitation. In 2010, the rate of foodborne camplylobacteriosis had decreased to 90.6 per 100000 (most likely estimates $58.5-109.7)^{4}$ (Figure 1).

When using human surveillance data, uncertainties arise due to underreporting of the true incidence of disease. Notified cases of illness and reported outbreaks represent a subset of all cases and outbreaks that occur. By using these data as indicators, it is assumed that they are representative of all the cases and outbreaks that occur in New Zealand. However, many cases do not visit a general medical practitioner or otherwise come to the attention of the medical system. It has been estimated that for every one notified case of gastrointestinal disease of infectious etilogy, there are $222\left(5^{\text {th }}\right.$ and $95^{\text {th }}$ percentiles 199-247) occuring in the community. ${ }^{11}$

Figure 1. Annual trend in estimated foodborne campylobacteriosis against five-year (2008-2012) goal, New Zealand ${ }^{4}$

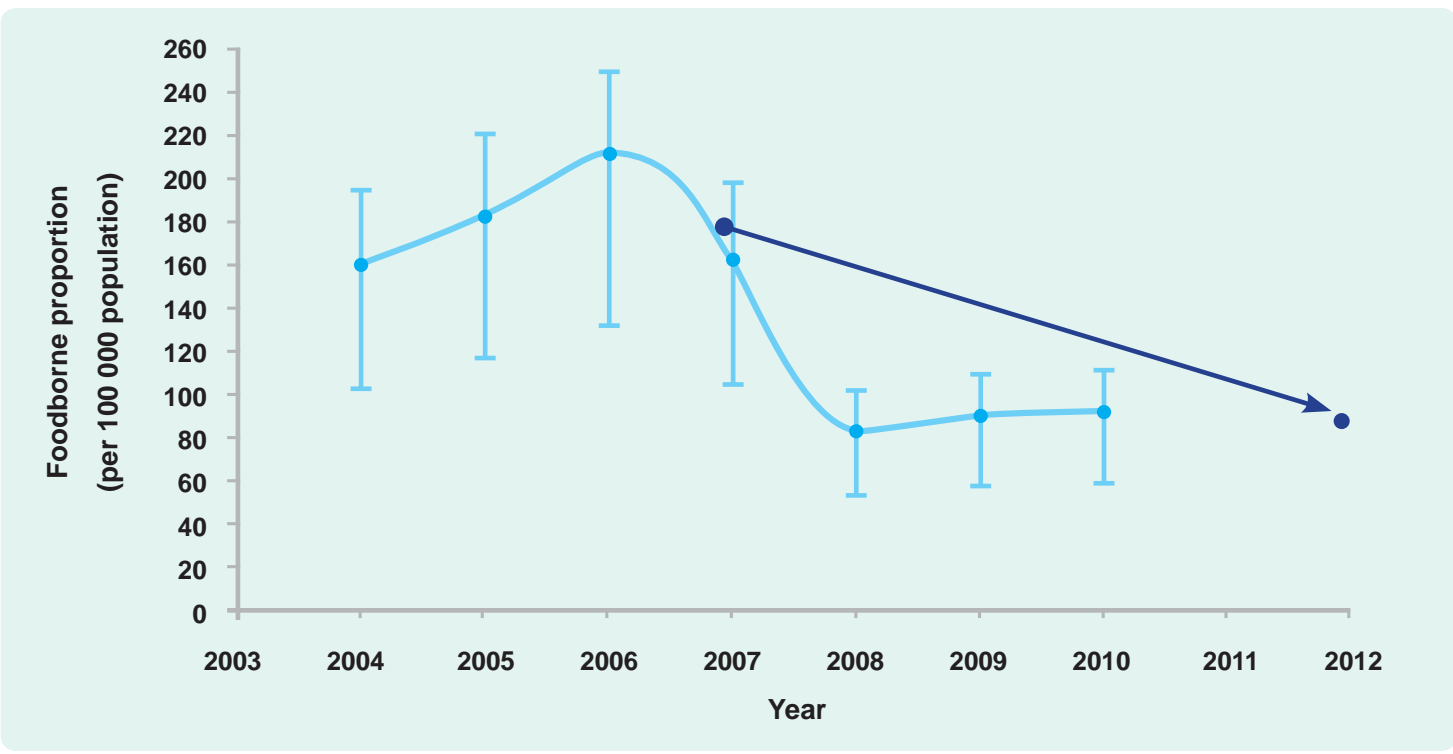

Note: The blue arrowed line represents the trend line from the baseline year (average of 2004-2007) to the five-year target (blue dot). 
In recent United States of America and United Kingdom studies the under-ascertainment for campylobacteriosis is estimated to be by factors of 30.3 and $9.5 .^{12,13}$ In addition, the estimates of the proportion of a disease that is foodborne have variable, wide confidence limits. ${ }^{9}$ These issues are not surprising as human disease surveillance systems are, in the main, control rather than strategy focused.

MAF has been fortunate to be able to integrate disease surveillance data with the poultry primary processing microbiological findings recorded in NMD. An association has been demonstrated between the decrease in Campylobacter carcass counts and the decline in human campylobacteriosis. This allowed the validation by statistical risk modelling of its Campylobacterreduction initiatives. It is rare to be able to substantiate such approaches in the real food safety world.

Surveillance of diseases that could be acquired from food has a pivotal role in informing all parts of MAF activities, from the development of its strategic priorities through to measurement of its agreed outputs and outcomes. The campylobacteriosis decline has been maintained, with there now being an estimated 70000 fewer cases each year overall in the community. ${ }^{4,11}$ There is good evidence that the Campylobacter Strategy has been responsible for a reduction in the annual number of cases of campylobacteriosis between 2006 and 2009 of some $53 \%$, with a saving of the order of US\$ 40 million annually. ${ }^{14}$

New Zealand is on course to meet its five-year campylobacteriosis reduction goal. However, the notified disease rate remains unacceptably high. Further work, such as a re-evaluation of the present Campylobacter poultry performance target, is ongoing, but initiatives to reduce the burden from non-foodborne sources are required also.

\section{Conflicts of interest}

None declared.

Funding

None.

\section{References:}

1. Langmuir AD. The surveillance of communicable diseases of national importance. New England Journal of Medicine, 1963, 268:182-192. doi:10.1056/NEJM196301242680405 pmid:13928666

2. New Zealand's Food Safety Risk Management Framework. Wellington, New Zealand Food Safety Authority, 2010 (http:// www.foodsafety.govt.nz/elibrary/industry/RMF full document -_11604_NZFSA_Risk_Management_Framework_3.1.pdf, accessed 15 March 2012).

3. Scientific Opinion of the Panel on Biological Hazards on a request from EFSA on Overview of methods for source attribution for human illness from foodborne microbiological hazards. European Food Safety Authority Journal, 2008, 764:1-43 (http://www.efsa.europa.eu/en/scdocs/doc/764.pdf, accessed 15 March 2012).

4. Institute of Environmental Science and Research Limited. Annual report concerning foodborne disease in New Zealand 2010. Wellington, Ministry of Agriculture and Forestry, 2011 (http:// www.foodsafety.govt.nz/elibrary/industry/FBI-report-2011.pdf, accessed15 March 2012).

5. Sears A et al. Marked campylobacteriosis decline after interventions aimed at poultry, New Zealand. Emerging Infectious Diseases, 2011, 17:1007-1015. doi:10.3201/eid1706.101272 pmid:21749761

6. Eberhart-Phillips $\mathrm{J}$ et al. Campylobacteriosis in New Zealand: results of a case-control study. Journal of Epidemiology and Community Health, 1997, 51:686-691. doi:10.1136/ jech.51.6.686 pmid:9519133

7. Wilson N. A systematic review of the aetiology of human campylobacteriosis in New Zealand 2005. Wellington, New Zealand Food Safety Authority, 2005 (http://www.foodsafety. govt.nz/elibrary/industry/Systematic_Review-Literature_Evidence. pdf, accessed 15 March 2012).

8. Mullner $\mathrm{P}$ et al. Molecular and spatial epidemiology of human campylobacteriosis: source association and genotype-related risk factors. Epidemiology and Infection, 2010, 138:1372-1383. doi:10.1017/S0950268809991579 pmid:20141645

9. Lake RJ et al. Risk ranking for foodborne microbial hazards in New Zealand: burden of disease estimates. Risk Analysis, 2010, 30:743-752. doi:10.1111/j.1539-6924.2009.01269.x pmid: 19645753

10. Biggs R. Beating the bacteria. Food New Zealand, 2012, February/ March:17-19.

11. Lake RJ et al. The disease pyramid for acute gastrointestinal illness in New Zealand. Epidemiology and Infection, 2010, 138:1468-1471. doi:10.1017/S0950268810000397 pmid:20196904

12. Scallan E et al. Foodborne illness acquired in the United Statesmajor pathogens. Emerging Infectious Diseases, 2011, 17:7-15. doi:10.3201/eid1701.09-1101p1 pmid:21192848

13. Tam CC et al.; IID2 Study Executive Committee. Longitudinal study of infectious intestinal disease in the UK (IID2 study): incidence in the community and presenting to general practice. Gut, 2012, 61:69-77. doi:10.1136/gut.2011.238386 pmid:21708822

14. Applied Economics. The economic cost of foodborne disease in New Zealand. Wellington, New Zealand Food Safety Authority, $2010 \quad$ (http://www.foodsafety.govt.nz/elibrary/ industry/economic-cost-foodborne-disease/index.htm, accessed 15 March 2012). 